\title{
Agrupamento Balanceado de Sujeitos a fim de Testar a Interface Gráfica de um Software de Geometria Interativa
}

\author{
Simone de Sousa Borges (sborges@icmc.usp.br) \\ Helena Macedo Reis (helenamcd@icmc.usp.br) \\ Luis Fernando de Souza Moro (luismoro@icmc.usp.br) \\ Vinicius Humberto Serapilha Durelli (durelli@icmc.usp.br) \\ Seiji Isotani (sisotani@icmc.usp.br) \\ Instituto de Ciências Matemáticas e de Computação - ICMC \\ Universidade de São Paulo - USP \\ São Carlos, Brasil
}

\begin{abstract}
Resumo. A interface é o principal mecanismo de comunicação entre o usuário e os recursos do sistema. No desenvolvimento de software educacional, projetos de interface de usuário elaborados apropriadamente podem minimizar o consumo da carga cognitiva dos usuários; assim, os usuários podem direcionar seus esforços para maximizar a compreensão dos conceitos de educação que estão sendo apresentados. Este artigo apresenta os resultados de um experimento no qual investigou-se como a gestão da memória de trabalho e o conhecimento anterior dos usuários, em informática e em geometria, podem influenciar no treinamento de usuários aprendendo a utilizar um software de geometria interativa. Foram aplicados três testes sobre conhecimentos específicos e realizado um experimento envolvendo sete estudantes de pós-graduação. Os resultados experimentais não indicaram diferenças significativas entre os alunos em relação aos escores obtidos nos testes.
\end{abstract}

Palavras-chave: geometria interativa, teoria da carga cognitiva, interface gráfica do usuário

\section{Towards Creating Balanced Groups of Subjects in Order to Test the GUI of an Interactive Geometry Software}

\begin{abstract}
The interface is the main mechanism of communication between user and system features. In educational software, properly designed user interfaces minimize the cognitive load on users; thereby users can direct their efforts to maximize their understanding of the educational concepts being presented. We investigated whether knowledge in computing, geometry and management of working memory affect the performance of a student during the learning process. To this end, we designed three tests about specific knowledge and carried out an experiment involving 7 postgraduate students. The experimental results indicated no significant differences between students in relation to the scores obtained on the tests.
\end{abstract}

Keywords: interactive geometry, cognitive theory load, graphical user interface 


\section{Introdução}

A comunicação entre um sistema computacional (software) e o ser humano ocorre por meio de uma interface, na qual o usuário pode introduzir dados de entrada para que o sistema, após o seu processamento, gere dados de saída. Interfaces projetadas de forma inapropriada (e.g., que não atendem a critérios mínimos de usabilidade), frequentemente prejudicam a forma como os usuários interagem com as funcionalidades do software (Nielsen, 1993). Ao realizar testes de usabilidade para elaborar uma interface, o desenvolvedor (i) maximiza as chances do usuário final utilizar o sistema de forma produtiva; (ii) viabiliza a rápida memorização das funcionalidades; e (iii) pode colaborar também para minimizar eventuais erros de interação.

No contexto de softwares educacionais para ensino de matemática/geometria, o desenvolvimento de interfaces também pode influenciar como os alunos exploram e aprendem os conceitos matemáticos/geométricos representados na tela do computador (Sedig \& Liang, 2006). Dentre os programas de computador para ensino de geometria com maior inserção no ambiente escolar temos os sistemas de geometria interativa (SGI). Estudos recentes têm sugerido que interfaces para SGI, que possuem elevado número de funções, apesar de serem úteis para usuários experientes, podem não ser adequadas para usuários iniciantes (Shimpf \& Spannagel, 2011). O usuário, ao utilizar uma tela com inúmeras funções, pode levar mais tempo para encontrar a função que necessita e, portanto, sua produtividade pode ser negativamente afetada. Apesar de alguns estudos terem abordado o desenvolvimento de interfaces para SGI, poucos trabalhos realizaram testes de usabilidade que focam tanto em problemas de interface quanto de aprendizagem (Kortenkamp \& Dohrmann, 2010; Mackrell, 2011). Devido a essa lacuna nas pesquisas, ainda não se definiu métricas para projetar interfaces adequadas para aprendizagem utilizando este tipo de software.

Neste mesmo cenário, estudantes que aprendem geometria com SGIs de baixa usabilidade, consomem grande parte de sua carga cognitiva aprendendo a utilizar o SGI. A carga cognitiva refere-se às cargas colocadas na memória de trabalho do aprendiz durante a instrução. Em muitos destes SGIs, o aluno direciona o uso desta carga, que é limitada, para aprender a utilizar a interface, afetando o aprendizado da matemática (Sedig \& Liang, 2006; Shimpf \& Spannagel, 2011). Portanto, os autores deste trabalho vêm pesquisando meios para aprimorar o entendimento de como as interfaces dos SGIs afetam a aprendizagem e como elas podem ser adaptadas para atender as necessidades dos alunos. Este experimento envolveu 7 alunos e 3 instrutores, utilizando um software de geometria interativa denominado iGeom (Isotani \& Brandão, 2008). Três habilidades foram avaliadas: (i) conhecimento em computação, (ii) conhecimento em geometria e a (iii) capacidade da memória de trabalho. Estas competências foram selecionadas pois é de interesse dos pesquisadores identificar o grau de influência destas habilidades em usuários não treinados, utilizando um SGI pela primeira vez.

O artigo encontra-se dividido da seguinte forma: na seção 2 são comentadas brevemente as Teorias da Carga Cognitiva e Memória de Trabalho. A seção 3 apresenta os Sistemas de Geometria Interativa. A seção 4 descreve o experimento elaborado e os resultados. E finalmente, nas seções 5 e 6 temos as ameaças à validade e a conclusões da pesquisa realizada, respectivamente.

\section{Teoria da Carga Cognitiva e Memória de Trabalho}

A carga cognitiva refere-se à demanda localizada na memória de trabalho dos alunos durante o processo de aprendizagem. Esse conceito é a base da teoria da carga cognitiva 
(TCC) (Paas et al., 2003). De acordo com essa teoria, os seres humanos possuem capacidade de processamento de informações limitada. Por exemplo, em geral, seres humanos apresentam dificuldades tanto para memorizar inúmeros conceitos apresentados em um curtos espaços de tempo como também em manter a atenção por longos períodos sem perder o foco. A TCC classifica a carga cognitiva dentro de três diferentes tipos: intrínseca, estranha e persistente (Schimpf \& Spannagel, 2011). A carga cognitiva intrínseca é relacionada com a complexidade da tarefa que precisa ser processada pelo aluno e não pode ser modificada pelo professor. Os desenvolvedores de interface se preocupam com a carga cognitiva estranha, pois ao apresentar elementos irrelevantes na tela, os alunos são obrigados a separar quais informações são importantes e quais não são para o seu aprendizado.

Diferentemente da carga intrínseca que é imutável, a carga cognitiva persistente pode ser controlada. Portanto, para projetar um sistema educacional que ajude os alunos a aprenderem a construir o seu conhecimento mais efetivamente, é importante que sejam criadas interfaces que reduzam a carga estranha e aumentem as cargas intrínseca e persistente. Interfaces que possuem mais funções, apesar de serem úteis para usuários experientes, podem mostrar-se demasiadamente complexas para os inexperientes. Isso pode indicar que a interface apresenta uma alta carga, consumindo considerável quantidade de memória de trabalho dos usuários.

\section{Sistemas de Geometria Interativa}

Sistemas de geometria interativa (SGI) implementam, na forma computacional, as ferramentas tradicionais usadas em sala de aula para ensinar geometria, tais como régua e compasso. O termo geometria refere-se a um ramo da matemática que estuda as propriedades e relações dos objetos geométricos (Isotani \& Brandão, 2008). Nesse contexto, estudantes que usam os SGIs podem criar representações abstratas dos objetos geométricos, medi-los e manipulá-los. Essas atividades permitem que os estudantes recebam um rápido feedback depois de manusear um objeto na tela. Consequentemente, estudantes podem testar conjecturas e hipóteses, encontrando novas relações e propriedades entre os objetos (Hollebrands, 2003).

Schimpf e Spannagel (2011) têm mostrado que uma das principais dificuldades reportadas pelos alunos enquanto aprendem como usar um SGI está relacionado à ampla variedade de funções nas interfaces. Um grande número de funções pode levar a ambiguidade, confusão dos estudantes com muitos detalhes, causar frustação e desmotivação. Deste modo, GUIs de SGI contendo muitos elementos gráficos podem impedir o processo de aprendizado, requerendo do usuário altas taxas de carga cognitiva. Como ressaltado por Sedig e Liang (2006), as habilidades cognitivas dos alunos são limitadas e devem ser direcionadas para ajudá-los a entender os conceitos matemáticos e não serem desperdiçadas no aprendizado de como utilizar a interface do software.

\section{Experimento: Planejamento e Execução}

Ao realizar um experimento anterior a este e que visava comparar duas versões de uma interface para um SGI denominado iGeom (Isotani \& Brandão, 2008), alguns resultados mostraram-se inclusivos. Apesar de os sujeitos selecionados serem todos estudantes universitários em um mesmo curso de graduação, o desempenho de um grupo mostrouse superior ao outro, sem motivo aparente (Reis et al., 2012). Inferiu-se que isto pode ter ocorrido devido aos diferentes níveis de conhecimento apresentados pelos sujeitos, onde 
estudantes com sólidos conhecimentos matemáticos concentraram-se em um grupo em detrimento do outro. Este experimento foi então planejado com o propósito de averiguar maneiras mais eficientes de distribuir os sujeitos da pesquisa em grupos balanceados a fim de se realizar novamente os testes com as diferentes versões do SGI.

\subsection{Planejamento}

Esta seção descreve a configuração e o planejamento usado no experimento a fim de se investigar uma possível relação entre as variáveis independentes capacidade da (i) memória de trabalho, conhecimentos em (ii) computação e (iii) geometria dos usuários e seu desempenho resolvendo exercícios de geometria utilizando um SGI. As seguintes questões de pesquisa (QP) foram elaboradas:

$\mathbf{Q P}_{\mathbf{1}}$ : Em termos de usabilidade, a capacidade de memorização por parte dos usuários influencia no aprendizado do software iGeom?

$\mathbf{Q P}_{2}$ : Em termos de usabilidade, o grau de proficiência dos usuários em Geometria influencia no aprendizado do software iGeom?

$\mathbf{Q P}_{3}$ : Em termos de usabilidade, o grau de proficiência dos usuários em computação influencia no aprendizado do software iGeom?

No contexto do experimento em questão, a usabilidade foi definida como as notas obtidas pelos sujeitos ao resolverem uma lista de exercícios utilizando o SGI iGeom pela primeira vez e sem treinamento prévio. Para a execução do experimento os seguintes passos foram executados: (i) questionário com questões pessoais, (ii) teste de conhecimentos básicos de computação, (iii) teste de conhecimentos de geometria e (iv) teste para avaliar a capacidade da memória de trabalho. Na primeira parte os sujeitos preencheram e solucionaram os três primeiros itens no período de uma hora, e na segunda hora, resolveram a lista de exercícios utilizando o SGI iGeom.

\section{Definição dos Objetivos}

As metas do experimento estão organizadas em cinco partes de acordo com o paradigma Goal/Question/Metric (GQM) (Wohlin et al., 2000): Objeto de Estudo, Objetivos, Perspectiva, Foco Qualitativo e Contexto.

Objeto de estudo: A interface de usuário de um software de Geometria Interativa chamado iGeom.

Objetivo: Avaliar a influência das variáveis independentes (i) conhecimento em geometria, (ii) habilidades computacionais, e (iii) capacidade da memória de trabalho na utilização de uma GUI para um SGI. O aprendizado de um novo software pode demandar considerável consumo da carga cognitiva do aprendiz, o que não é desejado. Usuários com alta capacidade de utilização de suas memórias de trabalho, em teoria, podem não ter dificuldades aprendendo a utilizar o iGeom ao mesmo tempo em que resolvem exercícios de geometria. Assim, usuários que demonstrem menor capacidade de manipulação da memória de trabalho podem ser sobrecarregados ao serem obrigados a realizar as duas tarefas ao mesmo tempo: aprender a utilizar o software e resolver as questões de geometria propostas.

Perspectiva: O experimento será executado do ponto de vista dos pesquisadores. 
Foco Qualitativo: O efeito primário investigado é a influência das variáveis independentes na utilização da GUI do iGeom mesurada pelas notas obtidas pelos sujeitos nos testes e questionários propostos.

Contexto: Estudantes de Pós-Graduação em Ciências da Computação com pouco ou nenhum conhecimento prévio sobre SGIs.

O experimento pode ser sumarizado pelo modelo abaixo (Wohlin et al., 2000):

\section{Analisar a GUI do software iGeom \\ para o propósito de avaliar \\ com respeito à usabilidade \\ do ponto de vista de pesquisadores \\ no contexto de estudantes de Pós-Graduação em Ciência da Computação com pouca ou nenhuma experiência utilizando SGIs.}

As questões de pesquisa $\mathbf{Q} \mathbf{P}_{1}, \mathbf{Q P}_{2}$ e $\mathbf{Q} \mathbf{P}_{3}$ foram separadas em três hipóteses a fim de viabilizar a aplicação de testes estatísticos. Cada hipótese foi testada em busca de evidências da possível correlação linear entre as variáveis independentes e a pontuação obtida pelos sujeitos utilizando o SGI. O primeiro par de hipóteses recebeu o nome de Memória de Trabalho, é identificada pelo acrônimo $\mathbf{M T}$ e se relaciona com $\mathbf{Q P}_{\mathbf{1}}$.

Hipótese Nula, $\mathbf{M T}_{\mathbf{0}}$ : Não há relação linear significativa entre a capacidade da memória de trabalho dos usuários e o aprendizado do iGeom (em termos das notas obtidas pelos sujeitos na resolução dos exercícios). Formalmente: $\mathbf{M T}_{\mathbf{0}}: \mathbf{r}=\mathbf{0}$

Hipótese alternativa, $\mathbf{M T}_{1}$ : Existe uma relação linear significativa entre a capacidade da memória de trabalho dos usuários e o aprendizado do iGeom (em termos das notas obtidas pelos sujeitos na resolução do teste). Formalmente: $\mathbf{M T}_{\mathbf{1}}: \mathbf{r} \neq \mathbf{0}$

O segundo par recebeu o nome de Conhecimento em Geometria (CG) e se relaciona com $\mathbf{Q P}_{2}$.

Hipótese Nula, $\mathbf{C G}_{\mathbf{0}}$ : Não há relação linear significativa entre o conhecimento de Geometria dos usuários e o aprendizado do iGeom (em termos das notas obtidas pelos sujeitos na resolução dos exercícios). Formalmente: $\mathbf{C G}_{\mathbf{0}}: \mathbf{r}=\mathbf{0}$

Hipótese alternativa, $\mathbf{C G}_{1}$ : Existe uma relação linear significativa entre 0 conhecimento de Geometria dos usuários e o aprendizado do iGeom (em termos das notas obtidas pelos sujeitos na resolução do teste). Formalmente: $\mathbf{C G}_{\mathbf{1}}: \mathbf{r} \neq \mathbf{0}$

E finalmente, o último par de hipóteses foi denominado Conhecimento Computacional (CC) e refere-se a $\mathbf{Q} \mathbf{P}_{3}$.

Hipótese Nula, $\mathbf{C C}_{0}$ : Não há relação linear significativa entre o conhecimento computacional dos usuários e o aprendizado do iGeom (em termos das notas obtidas pelos sujeitos na resolução dos exercícios). Formalmente: $\mathbf{C C}_{\mathbf{0}} \mathbf{:} \mathbf{r}=\mathbf{0}$

Hipótese alternativa, $\mathbf{C C}_{\mathbf{1}}$ : Existe uma relação linear significativa entre $\mathbf{o}$ conhecimento computacional dos usuários e o aprendizado do iGeom (em termos das notas obtidas pelos sujeitos na resolução do teste). Formalmente: $\mathrm{CC}_{1}: \mathbf{r} \neq \mathbf{0}$

\subsection{Design do Experimento}

Para verificar estas conjecturas, aplicou-se um modelo com fatores simples, com somente um tratamento para cada variável. As principais variáveis independentes foram 
memória de trabalho (MT), conhecimento computacional (CC) e conhecimento em geometria (CG) que foram comparados, alternadamente, à principal variável dependente que é a nota obtida pelos sujeitos ao resolver uma lista de exercícios utilizando o SGI. Esta variável foi denominada: iGeom. Com o objetivo de mesurar as habilidades dos sujeitos foram aplicados testes especialmente preparados para tal. O teste de conhecimentos computacionais aplicado consiste de questões simples relativas a tópicos básicos de informática. Os sujeitos receberam 2 pontos para cada questão correta e não pontuaram caso a resposta estivesse errada. Todas as questões foram de múltipla escolha não sendo possível atribuir avaliações parciais. $\mathrm{O}$ teste de geometria consistiu de 11 questões de múltipla escolha (letras a, b, c, d), desde o nível elementar até questões que demandavam maior conhecimento. A cada questão correta foi atribuído o valor 0,91 pontos.

O teste de memória de trabalho utilizado emprega a técnica conhecida como nback test (Dehn, 2008). Foi utilizado o site Cognitive Fun * que disponibiliza gratuitamente softwares que permitem realizar o n-back test. $\mathrm{O}$ teste consiste na apresentação de uma sequência de imagens, uma a uma, durante um período de tempo. O sujeito realizando o teste é, repetida e sequencialmente, convidado a responder se a figura que ele está visualizando no presente momento é igual a figura que foi apresentada " $n$ " figuras anteriores. Portanto, o teste pode ser configurado para testar a memória do sujeito, desde o nível 1 (considerado normal) até o nível 9 (extremamente difícil). Por questões de limitações do tempo envolvido para a aplicação do teste, ficou decidido que seria realizado o teste 2-back. Vale ressaltar que como o tempo de resposta é contabilizado no teste, quanto maior o resultado do 2-back test, pior o desempenho do sujeito, e vice-versa.

A Tabela 1 apresenta a pontuação dos sujeitos nos quatro testes. A Tabela 2, sumariza a pontuação apresentada na Tabela 1 . Uma análise inicial dos resultados indica uma grande flutuação nas notas. Podemos observar na Tabela 1 que dois sujeitos conseguiram resolver corretamente todos os exercícios propostos para o iGeom (sujeitos $\mathrm{B}$ e D), o que poderia indicar que talvez o teste estivesse muito simples, todavia o sujeito A pontuou 1,5 o que pode ser o indício de que ele teve dificuldades em resolvêlos.

Tabela 1. Pontuação dos sujeitos nos testes

\begin{tabular}{|c|c|c|c|c|}
\hline Sujeito & MT & CC & CG & iGeom \\
\hline A & 11,88 & 9,9 & 8,18 & 1,50 \\
\hline B & 9,80 & 9,8 & 6,36 & 10,00 \\
\hline C & 16,20 & 10 & 4,54 & 6,25 \\
\hline D & 9,60 & 9,9 & 8,18 & 10,00 \\
\hline E & 10,51 & 9,6 & 5,45 & 5,50 \\
\hline F & 25,83 & 9,8 & 6,36 & 7,00 \\
\hline G & 24,67 & 9,8 & 9,09 & 7,00 \\
\hline
\end{tabular}

\footnotetext{
${ }^{*}$ http://cognitivefun.net
} 
Tabela 2. Estatística descritiva sumarizando a pontuação dos sujeitos nos testes

\begin{tabular}{|l|c|c|c|c|}
\hline & MT & CC & CG & iGeom \\
\hline Min & 9,60 & 9,6 & 4,54 & 1,50 \\
\hline Max & 25,83 & 10,00 & 9,09 & 10,00 \\
\hline Média & 15,50 & 9,83 & 6,88 & 6,75 \\
\hline Mediana & 11,88 & 9,80 & 6,36 & 7,00 \\
\hline Desvio Padrão & 7,03 & 0,13 & 1,65 & 2,90 \\
\hline
\end{tabular}

\subsection{Análise dos Dados}

Esta seção sumariza os principais resultados observados com base no que foi descrito anteriormente. O coeficiente de correlação é uma medida da associação linear entre duas variáveis. O coeficiente de correlação calculado foi o de Spearman, que é um teste robusto de correlação não-paramétrico para determinar a intensidade com que duas variáveis estão linearmente associadas. $\mathrm{O}$ valor do coeficiente de correlação varia entre -1 e 1: em que 1 indica uma relação linear positiva perfeita, -1 representa uma relação linear negativa perfeita e 0 sinaliza que não há nenhuma relação linear. Os valores intermediários entre 0 e $|1|$ indicam a força da correlação. Na Tabela 3, a força da correlação é expressa usando o padrão "Guia Michelin" em cuja escala (* $p<0,05$, significativo; ** valor de $p<0,01$, altamente significativo; *** $p<0,001$, extremamente significativo). Quando não existe uma significância estatística, NS é usado e o valor $\boldsymbol{p}$ é dado entre parênteses. Os resultados foram agrupados de acordo com a questão de pesquisa:

Tabela 3. Estatística descritiva sumarizando os cálculos da correlação e da regressão

\begin{tabular}{|l|c|c|c|}
\hline & MT0, MT1 & CG0, CG1 & CC0, CC1 \\
\hline r (Spearman) & $-0,33$ & 0,23 & $-0,04$ \\
\hline r2 & $0,11(11 \%)$ & $0,05(5 \%)$ & 0,002 \\
\hline p-value & $\mathrm{NS}(0,89)$ & $\mathrm{NS}(0,94)$ & $\mathrm{NS}(1)$ \\
\hline
\end{tabular}

$\mathbf{Q P}_{1}$ - De acordo com o valor da correlação ( $r$ ) entre MT e os resultados obtidos na utilização do iGeom, nota-se que, quanto menores os resultados obtidos em MT, maior foi a nota obtida pelo sujeito durante a utilização do iGeom, Figura 1. A correlação entre as variáveis é fraca e negativa. O valor obtido para o $r 2$ indica que $11 \%$ da nota utilizando o iGeom talvez possa ser explicada pela interação com a capacidade da memória de trabalho do sujeito (MT). Entretanto, não é possível rejeitar a hipótese nula $\left(\mathrm{MT}_{0}\right)$ porque o valor para o $p$ ( $p$-value) é maior que 0,05 .

$\mathbf{Q P}_{2}$ - De acordo com o valor da correlação (r) entre CG e os resultados obtidos na utilização do iGeom, pode se notar que, aparentemente, quanto melhor o desempenho obtido em CG, maior foi a nota do sujeito durante a utilização do iGeom, Figura 2. De acordo com o valor calculado, a correlação entre as variáveis é fraca e positiva. O valor obtido para o $r 2$ indica que $5 \%$ da nota utilizando o iGeom talvez possa ser explicada pela interação com o conhecimento prévio em geometria por parte dos sujeitos (CG). Entretanto, não é possível rejeitar a hipótese nula $\left(\mathrm{CG}_{0}\right)$ porque o valor para o $p$ ( $p$-value) é maior que 0,05 . 


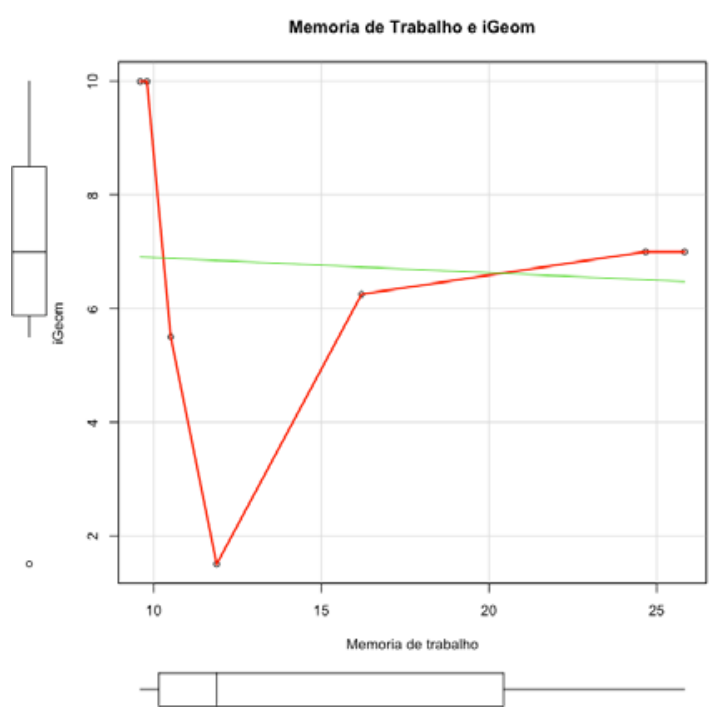

Figura 1. Relacionamento entre a Memória de Trabalho e nota do iGeom.

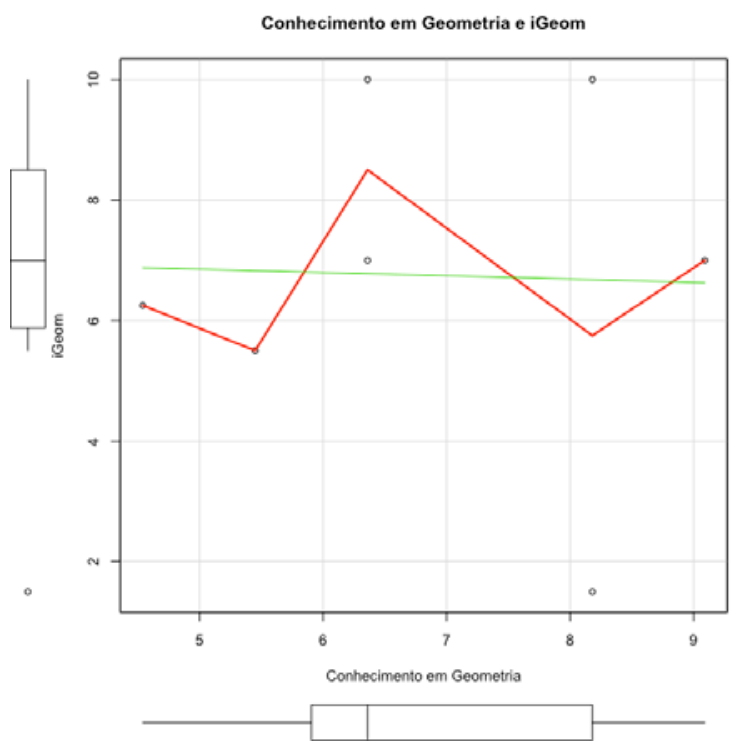

Figura 2. Relacionamento entre o Conhecimento em Geometria e nota do iGeom.

$\mathbf{Q P}_{3}$ - De acordo com o valor da correlação $(r)$ entre $\mathbf{C C}$ e os resultados obtidos na utilização do iGeom, observa-se na Tabela 2 que todos os sujeitos possuem alto conhecimento computacional, sendo que a nota mínima obtida foi 9,6 e a máxima 10. A média do grupo foi 9,83 com desvio padrão de apenas 0,13.

O valor calculado da correlação entre as variáveis foi muito pequeno, e indicou uma correlação fraca e negativa. Este resultado não era esperado uma vez que acreditava-se que quanto maior o conhecimento em computação maior seria a nota do iGeom. Atribuímos a instabilidade do resultado ao pequeno número de amostras/participantes, que resultou em um valor de $x$ concentrado entre 9,6 e 10 . Acredita-se que ao executar o experimento novamente com um número maior de sujeitos, este fenômeno tende a não se repetir. $O$ valor obtido para o $r 2$ não obteve significância estatística suficiente para afirmar que a nota utilizando o iGeom pode ser explicada pela interação com o conhecimento computacional dos sujeitos (CC). Além do que, não é possível rejeitar a hipótese nula $\left(\mathrm{CC}_{0}\right)$ porque o valor para o $p$ ( $p$-value) é maior que 0,05 . 


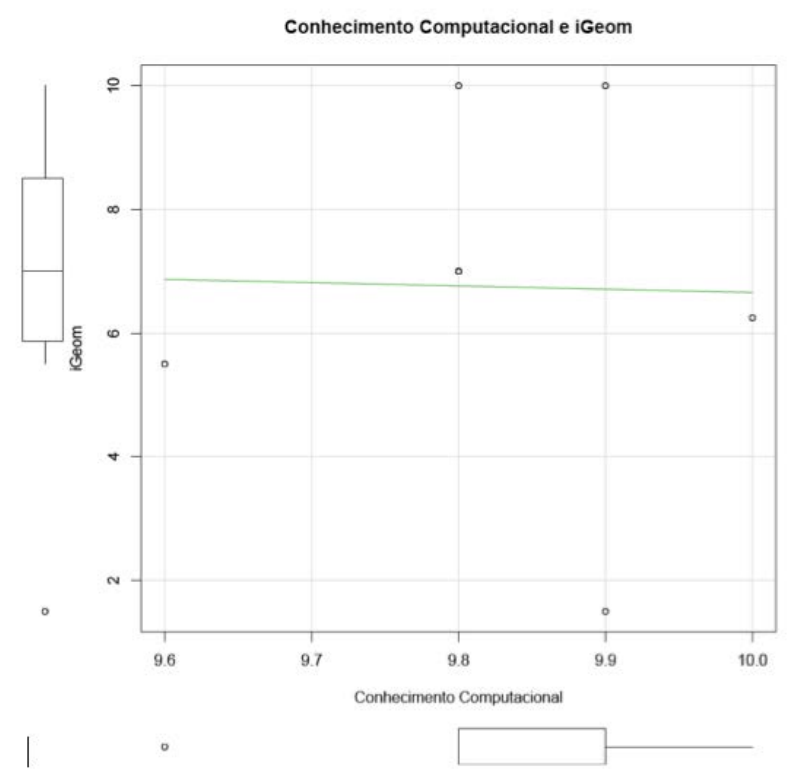

Figura 3. Relacionamento entre o Conhecimento em Computação e nota do iGeom.

\section{Ameaças à Validade}

Uma possível ameaça a validade externa, que está presente em quase todos os estudos de software, é a representatividade das amostras. Todos os sujeitos possuem curso superior e frequentam um curso de pós-graduação, portanto não se pode garantir que conclusões semelhantes seriam obtidas a partir dos resultados se tivéssemos usado uma população diferente. Também em relação à validade externa deste estudo, não é possível ter certeza de que esses resultados podem ser generalizados para toda a população de pós-graduandos em Ciência da Computação. Portanto, repetições deste estudo são necessárias para diferentes amostras da população. Pode-se dizer ainda que os dados do teste da memória de trabalho (n-back test) podem não ser representativos, uma vez que apenas uma etapa (2-back test) foi aplicada. Quanto as ameaças internas, existiu a preocupação em se garantir que o que foi avaliado foi a usabilidade da GUI do iGeom, entretanto a falta de conhecimentos em geometria pode ter impedido que alguns sujeitos completassem os exercícios propostos.

\section{Conclusão}

Pelos questionários e testes constatou-se que os sujeitos possuem avançados conhecimentos computacionais (o que era esperado) mas que todavia não foi possível afirmar se tal conhecimento é capaz de beneficiar usuários novatos na utilização do SGI iGeom. Aparentemente, o conhecimento prévio em geometria dos usuários pode ser um facilitador na aprendizagem da ferramenta. O conhecimento em geometria da maioria dos sujeitos estava acima da média das amostras, entretanto nenhum sujeito recebeu nota máxima no teste de geometria. Por outro lado, alguns sujeitos que conseguiram nota máxima resolvendo a lista de exercícios no iGeom, não tiveram desempenho semelhante na prova de geometria.

O dado mais expressivo e que parece exercer alguma influência no uso da interface por usuários iniciantes é capacidade da memória de trabalho dos usuários. Foi observado que $11 \%$ da nota utilizando o iGeom pode ter sido influenciada pelas habilidades de memorização dos usuários. Nenhuma técnica para eliminação ou normalização de outliers foi utilizada tendo em vista o número reduzido de participantes. 
Neste experimento foi utilizada somente uma execução do teste $n$-back no nível 2. Por esse motivo, para um próximo experimento planeja-se a execução do teste $n$-back configurado em diferentes níveis de dificuldade. Um outro benefício deste experimento foi a disponibilização de um pacote de replicação** que, acredita-se, possa ser utilizado por outros pesquisadores com propósitos acadêmicos ou mesmo investigando o mesmo assunto que foi tema do experimento. Os testes estatísticos mostraram-se inconclusivos, não sendo possível rejeitar nenhuma das hipóteses nulas formuladas devido aos altos valores de $p$ obtidos (todos próximos a 1). É possível inferir que o número pequeno de sujeitos presentes na amostra, apenas 7 indivíduos, pode ter influenciado nestes resultados. Como trabalhos futuros, pretende-se refinar o experimento desenvolvido, para viabilizar sua aplicação novamente, desta vez com um número maior de sujeitos que permita a coleta de dados estatisticamente significantes.

\section{Agradecimentos}

Os autores gostariam de agradecer ao CNPq pelo apoio financeiro (Proc. 383625/2012-2).

\section{Referências}

COYNE, J. T., BALDWIN, C., COLE, A., SIBLEY, C., \& ROBERTS, D. M. Applying Real Time Physiological Measures of Cognitive Load to Improve Training. In: Proceedings of the 5th International Conference on Foundations of Augmented Cognition. Neuroergonomics and Operational Neuroscience. Springer-Verlag, 2009, p. 469-478.

DEHN, J. M. Working Memory and Academic Learning: Assessment and Intervention. John Wiley \& Sons, 2008.

HOLLEBRANDS, K. F. High School Students' Understandings of Geometric Transformations in the Context of a Technological Environment. The Journal of Mathematical Behavior, 22(1), p.55-72, 2003.

ISOTANI, S.; BRANDÃO, L. O. An Algorithm for Automatic Checking of Exercises in a Dynamic Geometry System: iGeom. Computers \& Education, 51(3), p. 1283-1303, 2008.

KORTENKAMP, U.; DOHRMANN, C. User interface design for dynamic geometry software. Acta Didactica Napocensia, 3(2), p. 59-66, 2010.

MACKRELL, K. Design decisions in interactive geometry software. ZDM Mathematics Education, Springer, 43(3), p. 373-387, 2011.

NIELSEN, J. Usability Engineering. Boston: Academic Press Professional, 1993.

PAAS, F.; RENKLl, A.; SWELLER, J. Cognitive Load Theory and Instructional Design: Recent Developments. Educational Psychologist, 38(1):1-4, 2003.

REIS, H. M.; BORGES, S. S.; DURELLI, V. H. S.; MORO, L. F.; BRANDÃO, A. A. F.; BARBOSA, E. F.; BRANDÃO, L. O.; JAQUES, P. A.; BITTENCOURT, I. I.; ISOTANI, S. Towards Reducing Cognitive Load and Enhancing Usability Through a Reduced Graphical User Interface for a Dynamic Geometry System: An Experimental Study. In: Proceedings of the IEEE International Symposium on Multimedia (ISM). Irvine, CA. 2012, p. 445-450.

SCHIMPF, F.; SPANNAGEL, C. Reducing the graphical user interface of a dynamic geometry system. ZDM Mathematics Education, Springer, 43(3), 390-397, 2011.

SEDIG, K.; LIANG, H. N. Interactivity of Visual Mathematical Representations: Factors Affecting Learning and Cognitive Processes. Journal of Interactive Learning Research, 17(2), 179-212, 2006.

WOHLIN, C.; RUNESON, P.; HÖST, M.; OHLSOON, M. C.; REGNELL, B.; WESSLÉN, A. Experimentation in Software Engineering: An Introduction. Kluwer Academic Publishers, 2000.

** http://goo.gl/Om0hj 